\title{
Integrated Decision Algorithms for Auto-steered Electric Transmission System Asset Management
}

\author{
James McCalley, Vasant Honavar, Sarah Ryan, William Meeker, Daji Qiao, \\ Ron Roberts, Yuan Li, Jyotishman Pathak, Mujing Ye, and Yili Hong \\ Iowa State University, Ames, IA 50011, US \\ $\{j d m$, honavar, smryan, wqmeeker, daji, rroberts, tua, jpathak, \\ mye, hong\} @iastate.edu
}

\begin{abstract}
Electric power transmission systems are comprised of a large number of physical assets, including transmission lines, power transformers, and circuit breakers, that are capital-intensive, highly distributed, and may fail. Managing these assets under resource constraints requires equipment health monitoring integrated with system level decision-making to optimize a number of various operational, maintenance, and investment-related objectives. Industry processes to these ends have evolved ad-hoc over the years, and no systematic structures exist to coordinate the various decision problems. In this paper, we describe our progress in building a prototype structure for this purpose together with a software-hardware environment to deploy and test it. We particularly focus on the decision algorithms and the Benders approach we have taken to solve them in an integrated fashion.
\end{abstract}

Keywords: asset management, Benders decomposition, condition monitoring, decision algorithms, electric transmission, optimization, service-oriented architecture, software-hardware.

\section{Introduction}

There are three interconnected electric power transmission grids in North America: the eastern grid, the western grid, and Texas. Within each grid, power supplied must equal power consumed at any instant of time; also, power flows in any one circuit depend on the topology and conditions throughout the network. This interdependency means that should any one element fail, repercussions are seen throughout the interconnection, affecting system economic and engineering performance. Overall management requires decision in regards to how to operate, how to maintain, and how to reinforce and expand the system, with objectives being risk minimization and social welfare maximization. The three decision problems share a common dependence on equipment health or propensity to fail; in addition, their solutions heavily influence future equipment health. As a result, they are coupled, and optimality requires solution as a single problem. However, because network size (number of nodes and branches) together with number of failure states is so large, such a problem, if solved using traditional optimization methods, is intractable. In addition, the three decision problems differ significantly in decision-horizon, with operational decisions 
implemented within minutes to a week, maintenance decisions within weeks to a couple of years, and investment decisions within 2-10 years. Therefore, excepting the common dependence and effect on equipment health, the coupling is sequential, with solution to latter-stage problem depending on solution to former-stage problems. Because of this, the industry has solved them separately, with the coupling represented in a very approximate fashion via human communication mechanisms. We conjecture that resulting solutions are not only suboptimal, but they are not even very good solutions, a conjecture which motivates the work reported here.

A previous paper [1] described an initial design for a hardware-software prototype capable of auto-steering information-decision cycles inherent to managing operations, maintenance, and planning of the high-voltage electric power transmission systems. Section 2 of this paper describes a refined version of this overall design together with progress in implementing it. Section 3 summarizes the various optimization problems, providing problem statements when solved individually. Section 4 provides a new formulation, based on Benders decomposition, for a subgroup of problems, an approach that we eventually intend to apply to the entire set. Section 5 concludes.

\section{Overall Design and Recent Progress}

Figure 1 illustrates design of our prototype system for auto-steering informationdecision processes for electric transmission system asset management. This section overviews intended implementation and recent progress of the 5 different layers.

Layer 1, The power system: The prototype centers on a continuously running model of the Iowa power system using network data provided by a local utility company using a commercial-grade operator training simulator (OTS). The OTS is provided by ArevaT\&D (www.areva-td.com) and comprises the same energy management software system used by many major transmission control centers all over the world. The dataset on which this software system runs is the same dataset used by the utility company at their control center. This presents information security requirements that must be satisfied in our lab, since the data represents a critical national infrastructure. The work to implement this is intensive and is being supported under a cost-sharing arrangement between ArevaT\&D, ISU, and the utility company.

Layer 2, Condition sensors: Transformers are the most expensive single transmission asset, with typical costs between \$1-5M. The utility company has over 600 of them some of which have well exceeded their 40 year design life. All units undergo yearly dissolved gas-in-oil analysis (DGA) which, similar to a human blood test, provides information useful for problem diagnosis and prediction. We have obtained this data for all units and are using it to perform life prediction of the units. In addition, we are installing a real-time DGA monitor (www.kelman.co.uk) in one of the largest and oldest units and have been working on methods of transforming this data into health indicators that can be used in our decision algorithms.

Layer 3, Data communication and integration: The transformer monitor is equipped with a cellular modem provided by Cannon (www.cannontech.com) that communicates the real-time data to our lab. A federated data integration system has been designed to provide efficient, dependable, and secure mechanisms for interfacing Layer 4 data transformation algorithms with the data resources [2]. 
Layer 4, Data processing and transformation: The data available for equipment health prediction includes transformer monitoring and test data and weather/vegetation data which is useful for estimating probabilistic failure indices of transformers and overhead transmission lines [3].

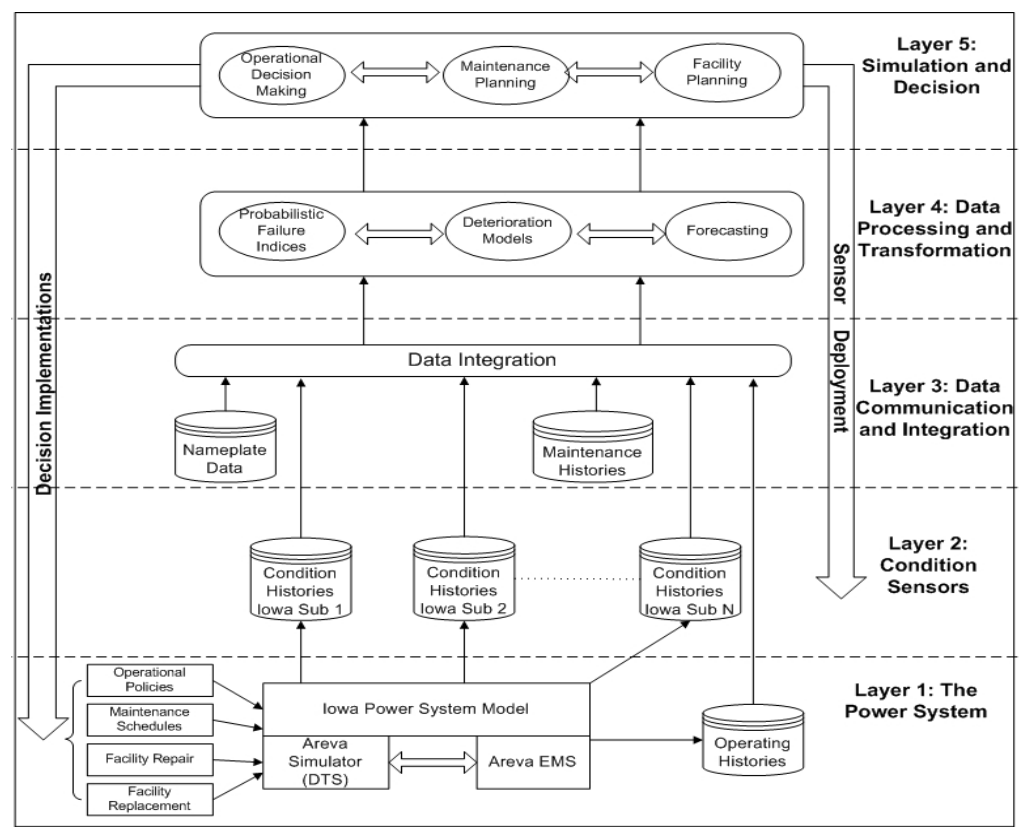

Fig. 1. Prototype system design

Layer 5, Simulation and decision: This layer utilizes probabilistic failure indices from layer 4 together with short and long-term system forecasts to drive integrated stochastic simulation and decision models. Resulting operational policies, maintenance schedules, and facility expansion plans are implemented on the power system (as represented by the ArevaT\&D simulator). The decision models are also used to discover the value of additional information. This valuation will be used to drive the deployment of new sensors and redeployment of existing sensors, impacting Layer 2. The integration of decision models is further described in Section 3.

A service-oriented architecture (SOA) is used for this software system. This framework, PSAM-s, for Power System Asset Management employs a Web servicesbased SOA. The core of the framework is the PSAM-s engine comprised of multiple services responsible for enabling interaction between users and other services that offer specific functionality. These services are categorized into internal services (part of the PSAM-s engine) and external services. The internal services include submission, execution, brokering, monitoring, and storage. The external services include data provision and information processing. These services and their overall architecture are illustrated in Fig. 2; additional description is provided in [4]. 


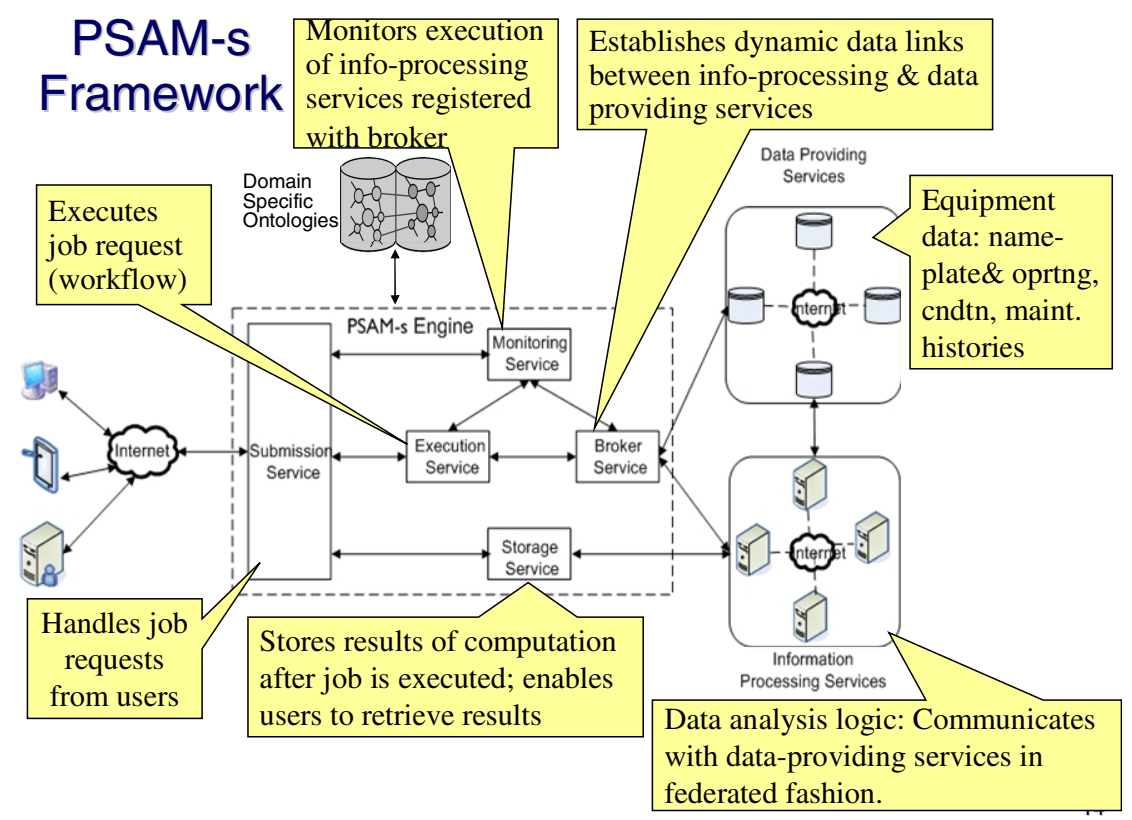

Fig. 2. A Service-Oriented Architecture

\section{Layer 5: Simulation, Decision and Information Valuation}

There are 6 basic risk-economy decision problems associated with power systems operation, maintenance, and planning, as illustrated in Table 1. The table illustrates the sequential coupling between the various problems in terms of information that is passed from one to the other. Information required to solve a problem is in its diagonal block and in the blocks left of that diagonal. Output information from solving a problem is below its diagonal block and represents input information for the lower-level problems. We briefly summarize each of these problems in what follows.

Operations: There are three operational sub-problems $[5,6]$.

- Unit commitment $(U C)$ : Given an hourly total load forecast over the next day or week, identify the hourly sequence of generation commitments (which generators are interconnected to the grid?) to maximize welfare (minimize costs) subject to the requirement that load must be satisfied, and also subject to physical limits on each generator associated with supply capability, start-up, and shut-down times.

- Optimal power flow (OPF): Given the unit commitment solution together with load requirements at each bus, and the network topology, determine the allocation of load to each generator and each generator's voltage set point to maximize social welfare, subject to Kirchoff's laws governing electricity behavior (encapsulated in a set of nonlinear algebraic "power flow" equations) together with constraints on branch flows, node voltages, and generator supply capability. 
Table 1. Summary of Power System Risk-Economy Decision Problems

\begin{tabular}{|c|c|c|c|c|c|c|c|}
\hline & \multicolumn{3}{|c|}{$\begin{array}{l}\text { Operations } \\
T=1-168 \text { hrs }\end{array}$} & \multicolumn{2}{|c|}{$\begin{array}{l}\text { Maintenance } \\
\mathrm{T}=1-5 \mathrm{yrs}\end{array}$} & \multirow{2}{*}{\begin{tabular}{|l}
$\begin{array}{c}\text { Planning } \\
\text { T=5-10 yrs }\end{array}$ \\
\end{tabular}} \\
\hline & & $\begin{array}{l}\text { Unit } \\
\text { commit } \\
\text { (UC) }\end{array}$ & $\begin{array}{l}\text { Optimal } \\
\text { power } \\
\text { flow } \\
\text { (OPF) }\end{array}$ & \begin{tabular}{|l|} 
Security \\
Assessmnt \\
(SA)
\end{tabular} & $\begin{array}{l}\text { Shortterm } \\
\text { maint }\end{array}$ & $\begin{array}{l}\text { Longterm } \\
\text { maint }\end{array}$ & \\
\hline \multirow{3}{*}{ 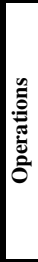 } & $\begin{array}{l}\text { Unit commit } \\
\text { (UC) }\end{array}$ & Total load & & & & & \\
\hline & $\begin{array}{l}\text { Optimal } \\
\text { power flow } \\
\text { (OPF) }\end{array}$ & \begin{tabular}{|l} 
Units \\
committed
\end{tabular} & $\begin{array}{l}\text { Bus loads, } \\
\text { topology }\end{array}$ & & & & \\
\hline & $\begin{array}{l}\text { Security } \\
\text { Assessment } \\
\text { (SA) }\end{array}$ & \begin{tabular}{|l|} 
Units \\
committed
\end{tabular} & $\begin{array}{l}\text { Operating } \\
\text { condition }\end{array}$ & $\begin{array}{l}\text { Weather, } \\
\text { failure data } \\
\text { inst. cndtn } \\
\text { data }\end{array}$ & & & \\
\hline \multirow{2}{*}{ 苋 } & $\begin{array}{l}\text { Shortterm } \\
\text { maint }\end{array}$ & \begin{tabular}{|l|} 
Units \\
committed
\end{tabular} & $\begin{array}{l}\text { Operating } \\
\text { condition }\end{array}$ & \begin{tabular}{|l|} 
Operating \\
(risk) \\
history
\end{tabular} & $\begin{array}{l}\text { Maint effcts, } \\
\text { failure data, } \\
\text { cdt history, } \\
\text { resources }\end{array}$ & & \\
\hline & $\begin{array}{l}\text { Longterm } \\
\text { maint }\end{array}$ & $\begin{array}{l}\text { Units } \\
\text { committed }\end{array}$ & $\begin{array}{l}\text { Operating } \\
\text { condition }\end{array}$ & \begin{tabular}{|l} 
Operating \\
(risk) \\
history
\end{tabular} & $\begin{array}{l}\text { ST maint } \\
\text { schedule, ST } \\
\text { eqp deter rate }\end{array}$ & $\begin{array}{l}\text { Cost of } \\
\text { capital, } \\
\text { failure data } \\
\text { cdt history }\end{array}$ & \\
\hline 葛 & $\begin{array}{l}\text { Investment } \\
\text { planning }\end{array}$ & \begin{tabular}{|l} 
Units \\
committed
\end{tabular} & $\begin{array}{l}\text { Operating } \\
\text { condition }\end{array}$ & \begin{tabular}{|l|} 
Operating \\
(risk) \\
history
\end{tabular} & \begin{tabular}{|l|} 
ST maint \\
schedule, ST \\
eqp. deter \\
rate
\end{tabular} & $\begin{array}{l}\text { LT maint } \\
\text { schedule, } \\
\text { LT eqp. } \\
\text { deter rate }\end{array}$ & $\begin{array}{l}\text { Cost of } \\
\text { capital, } \\
\text { failure data, } \\
\text { cdt history }\end{array}$ \\
\hline
\end{tabular}

- Security assessment (SA): Given the operating condition (which is economically optimal), find the best tradeoff between minimizing supply costs and minimizing risk associated with potential failures in the network. Presently, the industry solves this problem by imposing hard constraints on risk (or conditions associated with risk), thus obtaining a single objective optimization problem, but it is amendable to multiobjective formulation.

Maintenance: There are two maintenance-related sub-problems [7, 8].

- Short-term maintenance: Given a forecasted future operating sequence over an interval corresponding to a budget period (e.g., 1 year), together with a set of candidate maintenance tasks, select and schedule those maintenance tasks which most effectively reduce cumulative future risk, subject to resource (budget and labor) and scheduling constraints.

- Long-term maintenance: For each class of components, given a future loading forecast, determine an inspection, maintenance, and replacement schedule to maximize its operational reliability and its residual life at minimum cost. This multiobjective problem is typically addressed with the single objective to maximize residual life subject to constraints on operational reliability and cost.

Planning [9, 10]: Given a set of forecasted future load growths and corresponding operating scenarios, determine a network expansion plan that minimizes investment costs, energy production costs, and risk associated with potential failures in the network, subject to Kirchoff's laws together with constraints on branch flows, node voltages, and generator physical supply capabilities. This problem is often solved by minimizing investment and production costs while imposing constraints on risk. 


\section{Benders Decomposition and Illustration}

Benders decomposition is an appropriate method for problems that are sequentially nested such that solution to latter-stage problems depends on solution to former-stage problems. Mixed integer problems can be posed in this way as can stochastic programming problems. The operational problem described in Section 3, consisting of the sequence of UC, OPF, and SA, is both. To illustrate concepts, consider:

$$
\text { Min }: z=c(x)+d(y)
$$

$$
\begin{array}{lll}
\text { Problem } P \quad \text { s.t. } & A(x) \quad \geq b \\
& E(x)+F(y) \geq h
\end{array}
$$

This problem can be represented as a two-stage decision problem [11]:

Stage 1 (Master Problem): Decide on a feasible $x^{*}$ only considering (1a);

$$
\begin{aligned}
& \text { Min: } z=c(x)+\alpha^{\prime}(x) \\
& \text { s.t. } A(x) \quad \geq b
\end{aligned}
$$

where $\alpha^{\prime}(x)$ is a guess of stage 2 regarding stage 1 decision variable $x$, to be updated by stage 2 .

Stage 2 (Subproblem): Decide on a feasible $y^{*}$ considering (1b) given $x^{*}$ from stage 1 .

$$
\begin{aligned}
& \alpha\left(x^{*}\right)=\operatorname{Min} \quad d(y) \\
& \text { s.t. } \quad F(y) \geq h-E\left(x^{*}\right)
\end{aligned}
$$

The partition theorem for mixed-integer programming problems [12] provides an optimality rule on which Benders decomposition is based. If we obtain optimal solution $\left(z^{*}, x^{*}\right)$ in the first stage and then obtain optimal solution $y^{*}$ in the second stage, if $c\left(x^{*}\right)+d\left(y^{*}\right)=z^{*}$, then $\left(y^{*}, x^{*}\right)$ is the optimal solution for Problem P. The interaction between stages 1 and 2 is shown in Fig. 3 .

The procedure of Benders decomposition is a learning process (try-fail-tryinaccurate-try-...-solved). In the left part of Fig. 3, when the stage 1 problem is solved, the optimal value is then sent to stage 2 . Stage 2 problem has two steps: 1 )

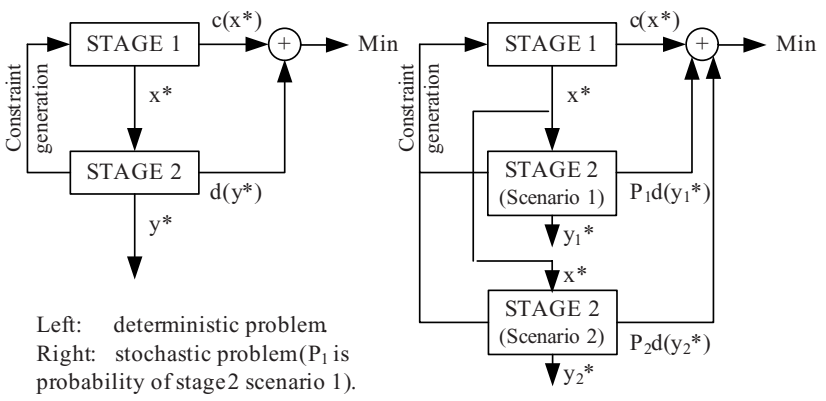

Fig. 3. Benders decomposition (modified from [11]) 
Check if the optimal solution from stage 1 is feasible. If it is not feasible, the stage 2 problem sends feasibility cuts back to stage 1 to be repeated under the additional constraints found in stage 2 to be in violation. 2) Check if the optimal guess of stage 2 from stage 1 is accurate enough. If it is not, a new estimation of $\alpha^{\prime}(x)$ is sent to stage 1. If the optimal rule is met, the problem is solved. This process is easily expanded to the stochastic programming case, as illustrated in the right part of Fig. 3 where the optimal value from stage 1 is sent to stage 2 , which has multiple scenarios. The process is exactly the same as the deterministic case, except that all constraint cuts and the optimal value from stage 2 are weighted by the probability of the scenario.

A 6-bus test system, Fig. 4, is used to illustrate. Generators are located at buses 1, 2, 6; loads at buses $3,4,5$. Possible contingencies considered include any failure of a single circuit. Detailed data for the system are provided in [5]. Figure 5 plots total cost of supply against time for a 24 hour period for two different scenarios: "average" uses contingency probabilities under normal weather, and " 10 *average" uses contingency probabilities under stormy weather. We observe in Fig. 5 the increased cost required to reduce the additional risk due to the stormy weather. Although the UC solution is the same in the two cases illustrated in Fig 5, it changes if the contingency probabilities are zero, an extreme situation which in fact corresponds to the way UC is solved in practice where UC and SA are solved separately. This is evidence that better solutions do in fact result when the different problems are solved together.

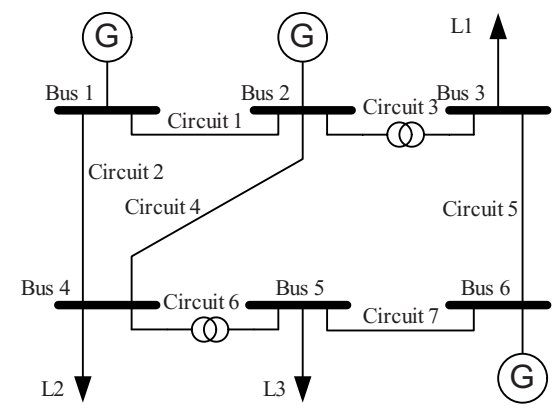

Fig. 4. 6-bus test system

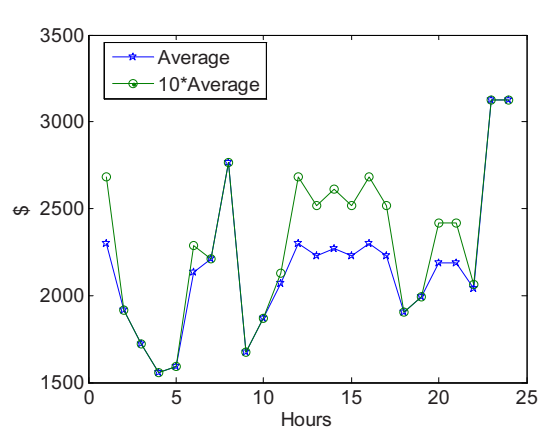

Fig. 5. Effect of contingency

\section{Conclusions}

Aging, capital intensive equipment comprise electric power grids; their availability largely determines the economic efficiency of today's electricity markets on which a nation's economic health depends; their failure results in increased energy cost, at best, and widespread blackouts, at worst. The balance between economy and reliability, or risk, is maintained via solution to a series of optimization problems in operations, maintenance, and planning, problems that traditionally are solved separately. Yet, these problems are coupled, and so solving them together necessarily improves on the composite solution. In this paper, we described a hardware-software system designed to address this issue, and we reported on our progress in developing this system, 
including acquisition of a real-time transformer monitor and of a commercial-grade power system simulator together with corresponding data modeling the Iowa power system. We also designed a service-oriented architecture to guide development of our software system. Finally, we implemented an optimization framework based on Benders decomposition to efficiently solve our sequential series of decision problems. This framework is promising; we expect it to be an integral part of our power system asset management prototype as we continue to move forward in its development.

\section{Acknowledgments}

The work described in this paper is funded by the National Science Foundation under grant NSF CNS0540293.

\section{References}

1. J. McCalley, V. Honavar, S. Ryan, W. Meeker, R. Roberts, D. Qiao and Y. Li, "Autosteered Information-Decision Processes for Electric System Asset Management," in Computational Science - ICCS 2006, 6th International Conference, Reading, UK, May 2831, 2006, Proceedings, Part III, Series: Lecture Notes in Computer Science , Vol. 3993, V. Alexandrov, G. van Albada, P. Sloot, J. Dongarra, (Eds.), 2006.

2. J. Pathak, Y. Jiang, V. Honavar, J. McCalley, "Condition Data Aggregation with Application to Failure Rate Calculation of Power Transformers," Proc. of the Hawaii International Conference on System Sciences, Jan 4-7, 2006, Poipu Kauai, Hawaii.

3. F. Xiao, J. McCalley, Y. Ou, J. Adams, S. Myers, "Contingency Probability Estimation Using Weather and Geographical Data for On-Line Security Assessment," Proc. of the 9th Int. Conf. on Probabilistic Methods Applied to Pwr Sys, June 11-15, 2006, Stockholm, Sweden.

4. J. Pathak, Y. Li, V. Honavar, J. McCalley, "A Service-Oriented Architecture for Electric Power Transmission System Asset Management," $2^{\text {nd }}$ International Workshop on Engineering ServiceOriented Applications: Design and Composition, Dec. 4, 2007, Chicago, Ill.

5. Y. Li, J. McCalley, S. Ryan, "Risk-Based Unit Commitment," to appear in Proc. of the 2007 IEEE PES General Meeting, June, 2007, Tampa Fl.

6. F. Xiao, J. McCalley, "Risk-Based Multi-Objective Optimization for Transmission Loading Relief Strategies," to appear, Proc. of the 2007 IEEE PES Gen Meeting, June, 2007, Tampa Fl.

7. J. McCalley, V. Honavar, M. Kezunovic, C. Singh, Y. Jiang, J. Pathak, S. Natti, J. Panida, "Automated Integration of Condition Monitoring with an Optimized Maintenance Scheduler for Circuit Breakers and Power Transformers," Final report to the Power Systems Engineering Research Center (PSerc), Dec., 2005.

8. Y. Jiang, J. McCalley, T. Van Voorhis, "Risk-based Maintenance Optimization for Transmission Equipment,” IEEE Trans on Pwr Sys, Vol 21, I 3, Aug. 2006, pp. 1191 - 1200.

9. J. McCalley, R. Kumar, O. Volij, V. Ajjarapu, H. Liu, L. Jin, W. Zhang, Models for Transmission Expansion Planning Based on Reconfigurable Capacitor Switching," Chapter 3 in "Electric Power Networks, Efficiency, and Security," John Wiley and Sons, 2006.

10. M. Ye, S. Ryan, and J. McCalley, "Transmission Expansion Planning with Transformer Replacement,” Proc. of 2007 Industrial Engr. Research Conf, Nashville, Tn, May 20-22, 2007.

11. S. Granville et al., "Mathematical decomposition techniques for power system expansion planning," Report 2473-6 of the Electric Power Research Institute, February 1988.

12. J. Benders, "Partitioning procedures for solving mixed-variables programming problems," Numerische Mathematik 4: 238-252, 1962. 